\title{
Recent ALICE results on ultra-peripheral collisions
}

\author{
David Horák* on behalf of the ALICE Collaboration \\ Faculty of Nuclear Sciences and Physical Engineering, \\ Czech Technical University in Prague, \\ Prague, Czech Republic \\ E-mail: david.horakecern.ch
}

The LHC is not only the most powerful collider for proton-proton and heavy-ion collisions, but it is also the most powerful source of photon collisions, because the protons and ions in the accelerator carry an electromagnetic field, which can be viewed as a flux of quasi-real photons. The photon-nucleus and photon-proton processes can be studied in ultra-peripheral collisions and offer an unique opportunity to study fundamental interactions in QED and QCD providing for example experimental data to search for saturation and shadowing effects. This text presents a summary of the latest $\rho^{0}$ and $\mathrm{J} / \psi$ cross section measurements with the ALICE detector.

7th Annual Conference on Large Hadron Collider Physics - LHCP2019

20-25 May, 2019

Puebla, Mexico

${ }^{*}$ Speaker. 


\section{Introduction}

The structure of a nucleon in perturbative QCD is described by a parton distribution function. It is well known that at low Bjorken- $x$ the proton structure is dominated by gluons [1]. It is natural to expect that for a fixed scale, the number of partons (quarks and gluons) in a nucleon cannot grow indefinitely. Their density will grow up to a point, where recombination will appear and balance it. An equilibrium, called saturation, is reached [2]. It is also an experimental fact that the structure of a free nucleon is different than that of a nucleon inside a nucleus, a depletion in the intermediate low x-region is observed which could be explained by mutual "shadowing" of the nucleons [3]. Recently, both these effects can be studied using ultra-peripheral collisions (UPC) at high-energy experimental facilities such as LHC or RHIC.

When two highly accelerated particles collide with an impact parameter $b$ larger than the sum of nuclear radii, the strong interaction is suppressed due to its short range and photon-induced interactions may occur. This is called an ultra-peripheral collision. The electromagnetic field of relativistic nuclei is contracted and its intensity (and the number of virtual photons surrounding the nucleus) is proportional to $Z^{2}$ as was already shown by Fermi in 1924 [4]. The physics of UPC is discussed at length in [5], while recent results at the LHC are reviewed in [6].

Vector meson photoproduction measurements in UPC is an interesting research field that can shed light on saturation and shadowing effects of QCD as cross sections of these processes are, in collinear leading order $\mathrm{PQCD}$, proportional to the gluon density squared [7].

From the experimental point of view, we are interested in events where only the vector meson is produced and nothing else is registered in the detector. In proton-nucleus collisions, the nucleus is the source of the photon and the proton is the target. The photon-proton centre-of-mass energy $W_{\gamma \mathrm{p}}$ is related to the rapidity of the vector meson: $W_{\gamma \mathrm{p}}^{2}=2 E_{\mathrm{p}} M_{\mathrm{VM}} \exp ( \pm y)$, where $E_{\mathrm{p}}$ is the beam target energy in the laboratory frame, $M_{\mathrm{VM}}$ is the vector meson rest mass and $y$ its rapidity.

The photoproduction of the vector meson off a proton can be elastic or dissociative. In the former, the proton remains in its ground state, in the latter, it gets excited and decays into a low mass state. In nucleus-nucleus collisions both particles can be a source or a target. The total cross section is then the sum of both cases. This interaction can be coherent, if the photon interacts with the whole nucleus, or incoherent where the photon interacts with one nucleon in the nucleus.

The ALICE detector $[8,9]$ is a powerful tool to study UPC thanks to the precise particle identification and precise momentum measurement at low transverse momentum in the central barrel. Moreover, the forward muon spectrometer provides a measurement of the $\mathrm{J} / \psi$ at high rapidities allowing a continuous measurement over a large rapidity range.

\section{Exclusive $\mathrm{J} / \psi$ photoproduction off protons}

The ALICE Collaboration has measured the energy dependence of exclusive photoproduction of $\mathrm{J} / \psi$ vector mesons off proton targets in ultra-peripheral $\mathrm{p}-\mathrm{Pb}$ collisions at a centre-of-mass energy per nucleon pair $\sqrt{s_{\mathrm{NN}}}=5.02 \mathrm{TeV}[10,11]$. Figure 1 shows all ALICE measurements for the exclusive photoproduction of $\mathrm{J} / \psi$ off protons as a function of $W_{\gamma \mathrm{p}}$. The data cover the $W_{\gamma \mathrm{p}}$ range from 24 to $706 \mathrm{GeV}$, which corresponds to three orders of magnitude in $x$, from $2 \times 10^{-2}$ to $2 \times 10^{-5}$. 


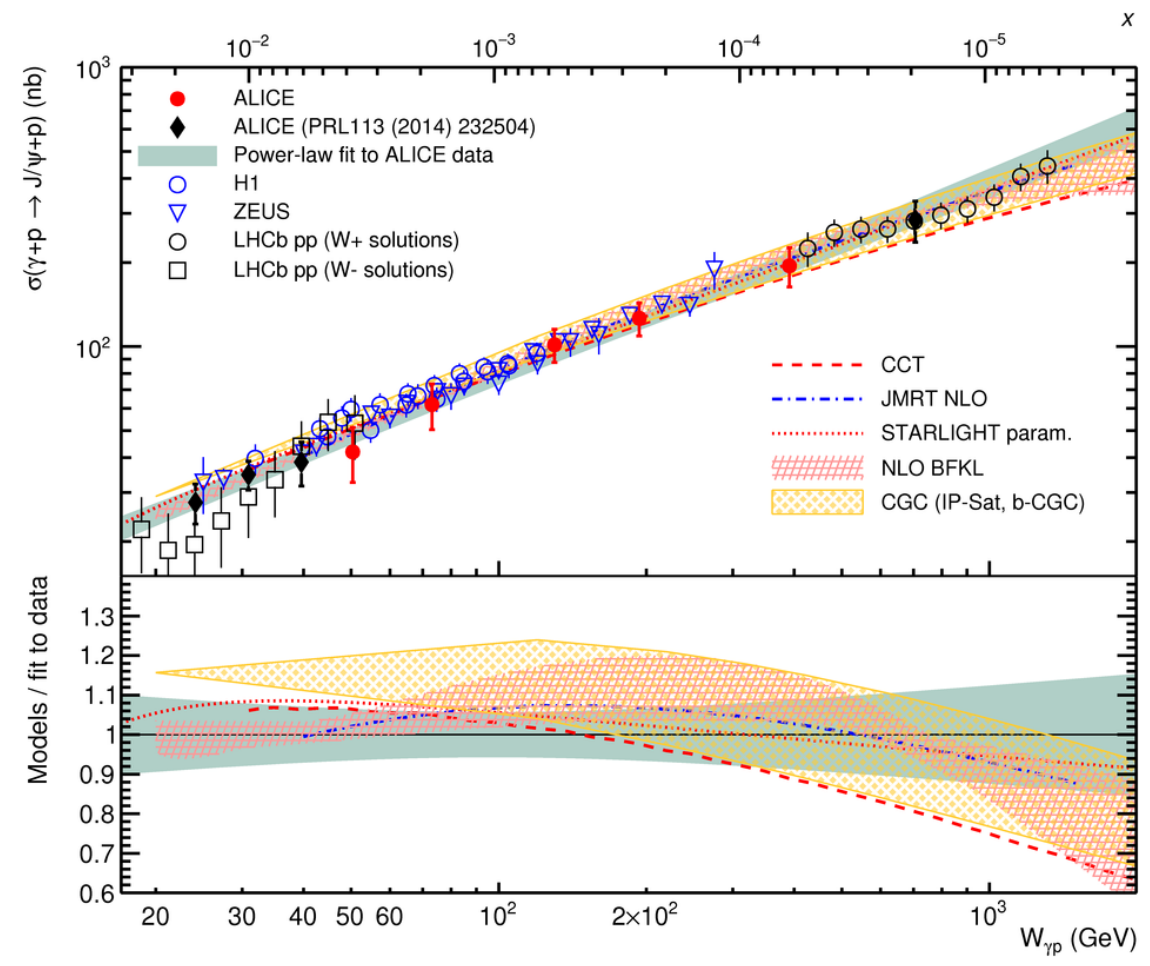

Figure 1: (Upper panel) ALICE data (black and red symbols) on exclusive photoproduction of $J / \psi$ off protons as a function of the centre-of-mass energy of the photon-proton system $W_{\gamma \mathrm{p}}$, obtained in UPC of protons and lead nuclei at $\sqrt{s_{\mathrm{NN}}}=5.02 \mathrm{TeV}$, compared to a power-law fit, to data from HERA, to the solutions from $\mathrm{LHCb}$ and to theoretical models. The uncertainties are the quadratic sum of the statistical and systematic uncertainties. (Lower panel) Ratio of the models shown in the upper panel to the power-law fit through the ALICE data points. The Bjorken- $x$ value corresponding to $W_{\gamma p}$ is also displayed on the top of the figure. Taken from [10].

The comparison of ALICE measurements with data from other experiments as well as to the results from different models is also shown in Fig. 1. HERA and ALICE data are compatible within uncertainties. LHCb measured the exclusive production of $\mathrm{J} / \psi$ in pp collisions [12], where the photon source can not be identified. They reported two solutions which also agree with ALICE measurements. The theoretical models are also in reasonable agreement with our data. The CCT model [13] is based on the colour dipole model and an energy-dependent hot-spot structure of the proton in the impact-parameter plane. The JMRT group [14] uses the computation from M. Ryskin [7] and includes also next-to-leading (NLO) contributions. STARlight [15] uses a power-law fit of fixed-target and HERA data. The last two models describe data using colour-dipole model and the colour glass condensate [16] and the NLO BFKL [17] evolution of HERA data.

\section{Vector meson photonuclear production in $\mathrm{Pb}-\mathrm{Pb} \mathrm{UPC}$}

ALICE performed the first measurement of the cross section for coherent photoproduction of $\rho^{0}$ vector mesons in $\mathrm{Pb}-\mathrm{Pb}$ collisions at $\sqrt{s_{\mathrm{NN}}}=2.76 \mathrm{TeV}$ [18]. The $\rho^{0}$ is reconstructed using the $\pi^{+} \pi^{-}$decay channel in the rapidity range $|y|<0.5$. The rapidity interval corresponds to a 

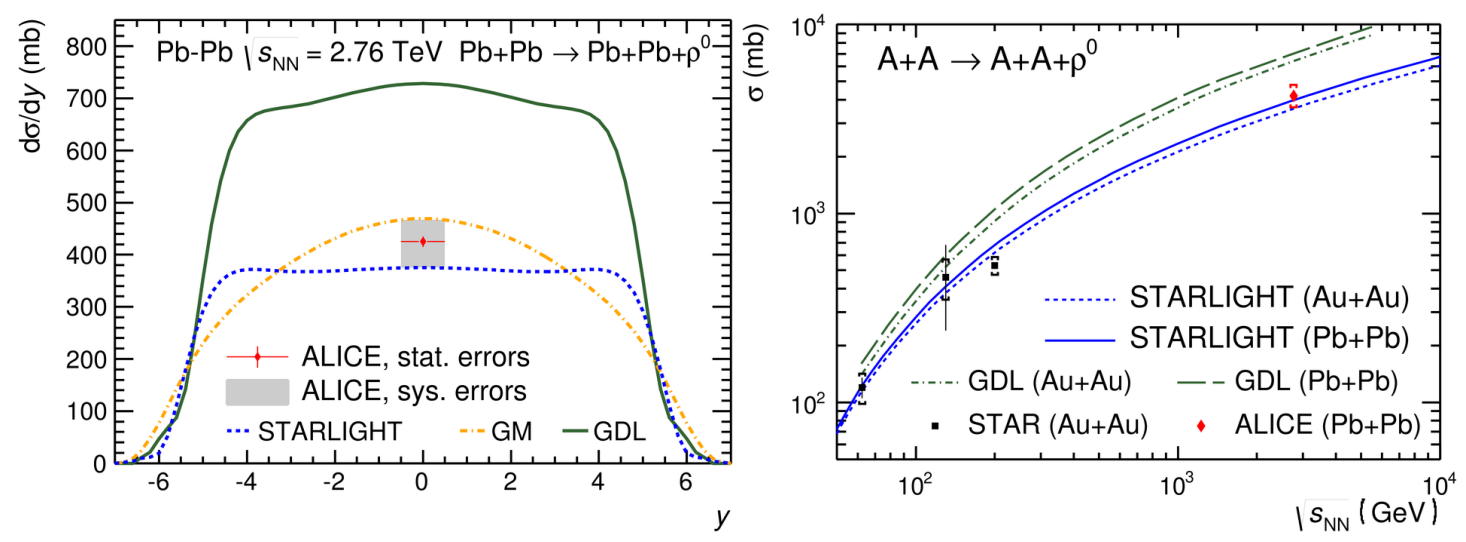

Figure 2: Left: The cross section for coherent photoproduction of $\rho^{0}, \mathrm{~d} \sigma / \mathrm{d} y$, in ultra-peripheral collisions for the three models compared to the ALICE result. Right: Total cross section for coherent and exclusive $\rho^{0}$ production. The results from ALICE and STAR are compared to the STARLIGHT and GDL predictions for $\mathrm{Pb}-\mathrm{Pb}$ and $\mathrm{Au}-\mathrm{Au}$. Taken from [18].

$\gamma$-nucleon centre-of-mass energy in the range $36 \leq W_{\gamma \mathrm{Pb}} \leq 59 \mathrm{GeV}$, about a factor of 4 higher than in any previous measurement. The measurement of coherent $\rho^{0}$ photoproduction probes the dynamics of QCD at a semi-hard scale and due to the large cross section offers a possibility to study the approach to the black disk limit of QCD. The coherent $\rho^{0}$ photoproduction cross section, $\mathrm{d} \sigma / \mathrm{d} y$, is shown and compared to model predictions in Fig. 2. The measured cross section is in agreement with STARlight [15], which is based on the Vector Meson Dominance model, however it neglects the elastic part of the $\rho^{0}$-nucleon cross section. The calculation by Gonçalves and Machado (GM) [19] uses a Colour Dipole Model and it also is in agreement with data. The last model, GDL (Glauber-Donnachie-Landshoff) [20] is a factor of 2 higher than data.

ALICE will also measure coherent $\rho^{0}$ photoproduction in Run 2 at $\sqrt{s_{\mathrm{NN}}}=5.02 \mathrm{TeV}$. A larger data sample in Run 2 will enable to observe the diffractive dip in the transverse momentum spectrum and the kink the invariant mass spectrum caused by the interference with the $\omega$ vector meson make it possible to measure its contribution. Measurement of the rapidity dependence with combination of forward neutron classes will allow us to test the method proposed in [21].

The ALICE Collaboration has measured the $\mathrm{J} / \psi$ photoproduction at forward and mid-rapidity in ultra-peripheral $\mathrm{Pb}-\mathrm{Pb}$ collisions in Run 1 at $\sqrt{s_{\mathrm{NN}}}=2.76 \mathrm{TeV}[22,23]$. The $\mathrm{J} / \psi$ is identified via its leptonic decay. The measurement at mid-rapidity allows the exploration of the region $x=\left(M_{\mathrm{J} / \psi} / \sqrt{s_{\mathrm{NN}}}\right) \exp ( \pm y) \approx 10^{-3}$ at a scale about $M_{\mathrm{J} / \psi}^{2} / 4 \mathrm{GeV}^{2}$, where the uncertainty in the nuclear gluon shadowing distribution is large. The results are compared to theoretical models in Fig. 3. Models based on moderate nuclear shadowing, as the AB-EPS09 model [24], provide a good description of data.

ALICE reported a measurement of the coherent photoproduction of $\mathrm{J} / \psi$ in ultra-peripheral $\mathrm{Pb}-\mathrm{Pb}$ collisions at Run 2 at a centre-of-mass energy $\sqrt{s_{\mathrm{NN}}}=5.02 \mathrm{TeV}$ [25]. The $\mathrm{J} / \psi$ was detected via its dimuon decay in the forward rapidity region of the detector. The increase in luminosity, plus advances in the trigger efficiency produced an approximately 200 fold increase in the data-sample size with respect to the Run 1 results discussed in the previous paragraph and shown in Fig. 3. The cross section is shown (Fig. 4) in six rapidity bins, covering the interval $-4.0<y<-2.5$. These 


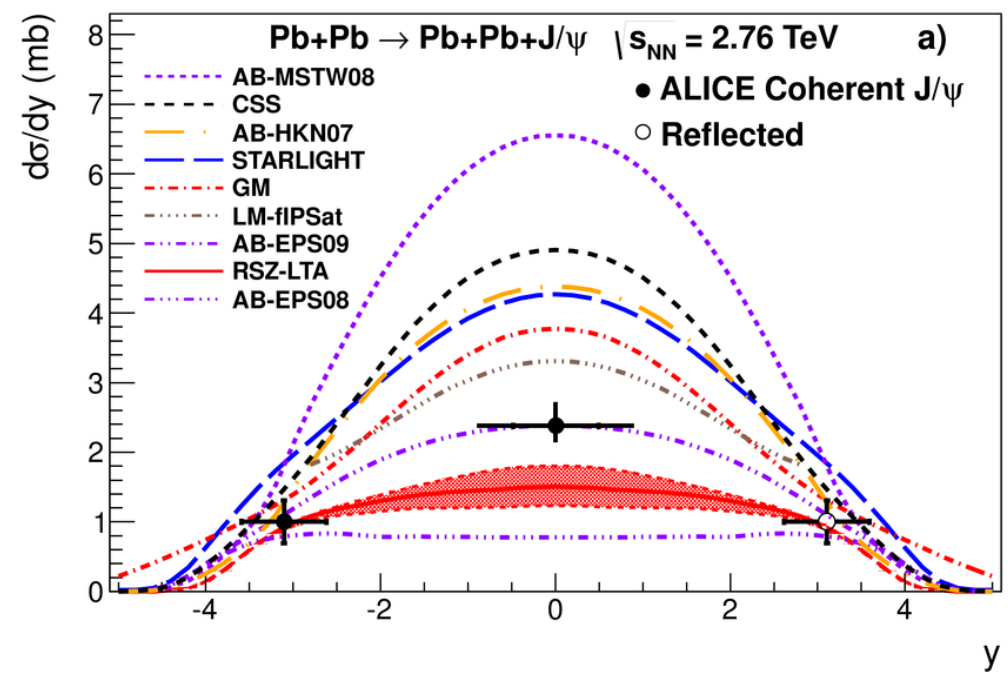

Figure 3: The cross section for coherent photoproduction of $\mathrm{J} / \psi, \mathrm{d} \sigma / \mathrm{d} y$, at $\sqrt{s_{\mathrm{NN}}}=2.76 \mathrm{TeV}$ compared to theoretical models. Taken from [22].

results are compared to various theoretical models. The Impulse Approximation is based on the data from the exclusive $\mathrm{J} / \psi$ photoproduction off protons and neglects all nuclear effects except for coherence and overpredicts data by almost a factor of two showing that moderate gluon shadowing effects play a role in the photoproduction process. STARlight [15] also contains no nuclear shadowing effects and also overestimates data. Two calculations from GKZ [26] based on EPS09 and Leading Twist Approximation (LTA) describe the data only in the forward region. Predictions by Gonçalves and Machado (GM) [27] based on IIM and b-CGC models for the scattering amplitude underpredict the data. Predictions by Lappi and Mantysaari (LM) [28] based on the IP-sat model give reasonable agreement throughout the range of the prediction. Calculations from Luszczak and Schafer (LS) [29] within the colour-dipole formulation of the Glauber-Gribov theory are in agreement with data. Cepila, Contreras and Krelina (CCK) [30] prediction based on the extension of the energy dependent hot-spot model agrees with data at most forward rapidities, but underpredicts them at semi-forward rapidities. The IPsat (LM) and BGK-I (LS) predictions, based on the colour-dipole model and a saturation prescription, describe best the measured cross section.

ALICE also reported the measurement of an excess in the yield of $\mathrm{J} / \psi$ at very low transverse momentum $\left(p_{\mathrm{T}}<0.3 \mathrm{GeV} / c\right)$ in peripheral hadronic $\mathrm{Pb}-\mathrm{Pb}$ collisions at $\sqrt{s_{\mathrm{NN}}}=2.76 \mathrm{TeV}$ [31]. Remarkably, the measured nuclear modification factor of $\mathrm{J} / \psi$ in the rapidity range $2.5<y<4$ reaches about 7 (2) in the $p_{\mathrm{T}}$ range $0-0.3 \mathrm{GeV} / \mathrm{c}$ in the $70-90 \%(50-70 \%)$ centrality class as is shown in Fig. 5. The $\mathrm{J} / \psi$ production cross section associated with the observed excess is obtained under the hypothesis that coherent photoproduction of $\mathrm{J} / \psi$ is the underlying physics mechanism. This measurement and the UPC-one described above have been used to extract the photonuclear cross section and the nuclear modification factor down to $x \approx 4 \cdot 10^{-5}$ [32]. The appearance of photon-induced hard process in hadronic $\mathrm{Pb}-\mathrm{Pb}$ collisions opens new theoretical and experimental challenges and opportunities, see e.g. [33,34]. In particular, coherent photoproduction accompanying hadronic collisions may provide insight into the dynamics of photoproduction and nuclear 


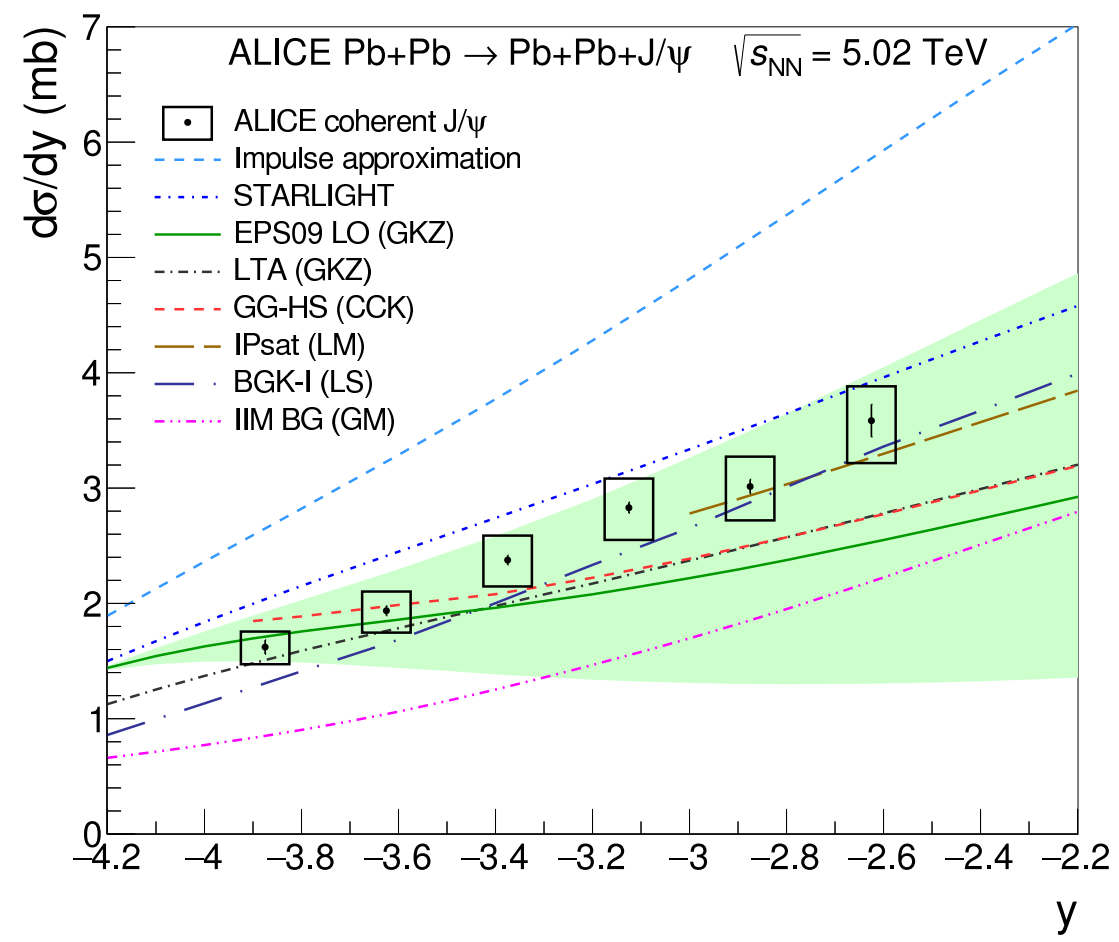

Figure 4: The differential cross section, $\mathrm{d} \sigma / \mathrm{d} y$, for coherent photoproduction of $\mathrm{J} / \psi$ at $\sqrt{s_{\mathrm{NN}}}=5.02 \mathrm{TeV}$ compared to theoretical models. The error bars represent the statistical uncertainties, the boxes around the points the systematic uncertainties. The green band represents the uncertainties of the EPS09 LO calculation. Taken from [25].

reactions, as well as become a novel probe of the quark-gluon plasma.

\section{Conclusions and outlook}

As shown in the previous sections, ALICE is an excellent detector to investigate QCD using UPC. We measured exclusive $\mathrm{J} / \psi$ production off protons in a range of $\gamma$ p energies from 20 to 700 $\mathrm{GeV}$ in Run 1. With Run 2 data these measurements will reach well above $1 \mathrm{TeV}$. ALICE studies the approach to the black-disk limit of QCD with coherent $\rho^{0}$ production in $\mathrm{Pb}-\mathrm{Pb}$ UPC.

Models containing moderate shadowing effect showed a consistency with the measurement of coherent $\mathrm{J} / \psi$ photoproduction in forward and mid-rapidity. The future data from Run 3 and Run 4 are expected to be a powerful tool to access the gluon structure functions down to $x=10^{-5}$ [35]. Acknowledgments: This work has been partially supported by the grant 18-07880S of the Czech Science Foundation (GACR).

\section{References}

[1] H. Abramowicz et al. [H1 and ZEUS Collaborations], Combination of measurements of inclusive deep inelastic $e^{ \pm} p$ scattering cross sections and QCD analysis of HERA data, Eur. Phys. J. C 75 (2015) no.12, 580 doi:10.1140/epjc/s10052-015-3710-4 [arXiv:1506.06042 [hep-ex]]. 


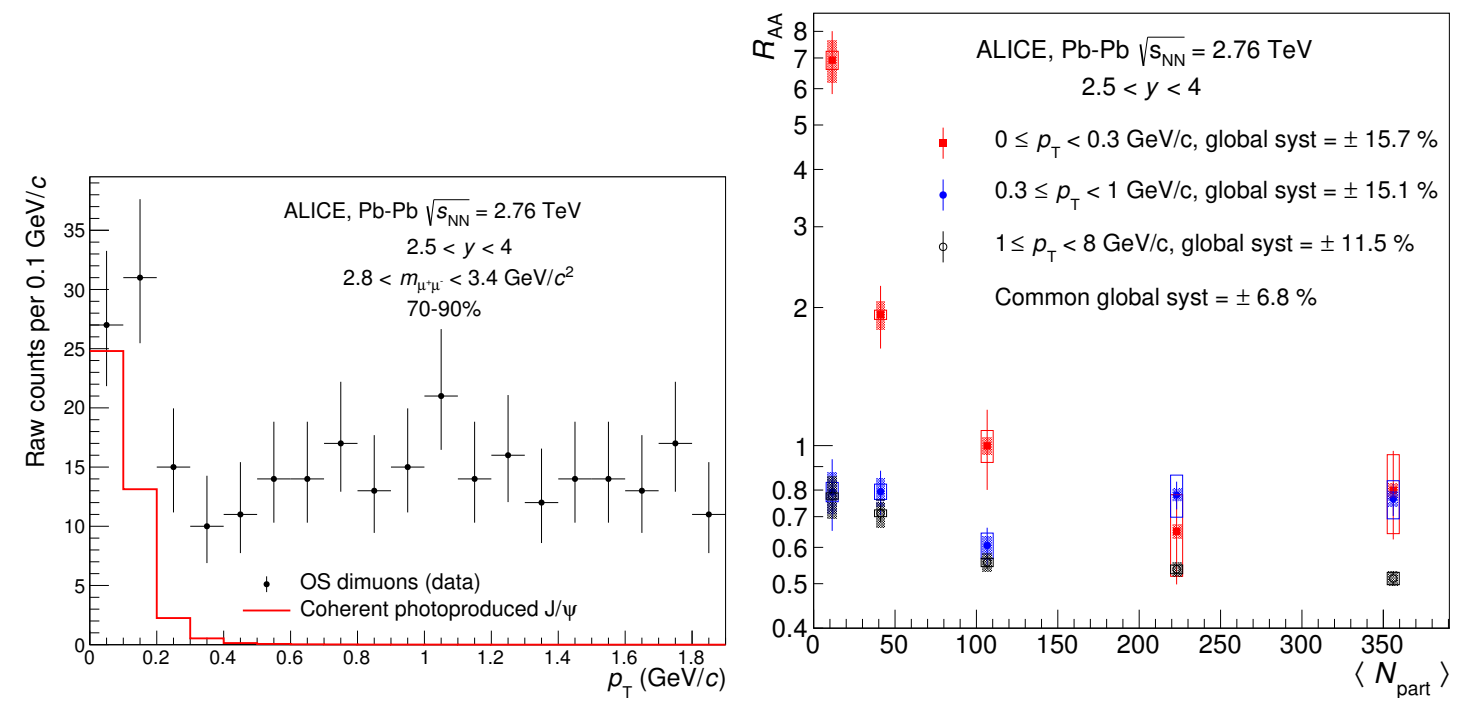

Figure 5: Left: Raw opposite-sign dimuon $p_{\mathrm{T}}$ distribution for the invariant mass range $2.8<m\left(\mu^{+} \mu\right)<3.4$ $\mathrm{GeV} / c^{2}$ and centrality class $70-90 \%$. The red line represents the $p_{\mathrm{T}}$ distribution of coherently photoproduced $\mathrm{J} / \psi$ as predicted by the STARLIGHT MC generator in $\mathrm{Pb}-\mathrm{Pb}$ ultra-peripheral collisions and convoluted with the response function of the muon spectrometer. Right: $\mathrm{J} / \psi R_{\mathrm{AA}}$ for $3 p_{\mathrm{T}}$ ranges in $\mathrm{Pb}-\mathrm{Pb}$ collisions at $\sqrt{s_{\mathrm{NN}}}=2.76 \mathrm{TeV}$. Taken from [31].

[2] L. V. Gribov, E. M. Levin and M. G. Ryskin, Semihard Processes in QCD, Phys. Rept. 100 (1983) 1. doi:10.1016/0370-1573(83)90022-4

[3] N. Armesto, Nuclear shadowing, J. Phys. G 32 (2006) R367 doi:10.1088/0954-3899/32/11/R01 [hep-ph/0604108].

[4] E. Fermi, On the theory of collisions between atoms and electrically charged particles, Nuovo Cim. 2 (1925) 143 doi:10.1007/BF02961914, 10.1142/97898127042140026 [hep-th/0205086].

[5] A. J. Baltz et al., The Physics of Ultraperipheral Collisions at the LHC, Phys. Rept. 458 (2008) 1 doi:10.1016/j.physrep.2007.12.001 [arXiv:0706.3356 [nucl-ex]].

[6] J. G. Contreras and J. D. Tapia Takaki, Ultra-peripheral heavy-ion collisions at the LHC, Int. J. Mod. Phys. A 30 (2015) 1542012. doi:10.1142/S0217751X15420129

[7] M. G. Ryskin, Diffractive J / psi electroproduction in LLA QCD, Z. Phys. C 57 (1993) 89. doi:10.1007/BF01555742

[8] K. Aamodt et al. [ALICE Collaboration], The ALICE experiment at the CERN LHC, JINST 3 (2008) S08002. doi:10.1088/1748-0221/3/08/S08002

[9] B. B. Abelev et al. [ALICE Collaboration], Performance of the ALICE Experiment at the CERN LHC, Int. J. Mod. Phys. A 29 (2014) 1430044 doi:10.1142/S0217751X14300440 [arXiv:1402.4476 [nucl-ex]].

[10] S. Acharya et al. [ALICE Collaboration], Energy dependence of exclusive J/ $\psi$ photoproduction off protons in ultra-peripheral p-Pb collisions at $\sqrt{s_{\mathrm{NN}}}=5.02 \mathrm{TeV}$, Eur. Phys. J. C 79 (2019) no.5, 402 doi:10.1140/epjc/s10052-019-6816-2 [arXiv:1809.03235 [nucl-ex]].

[11] B. B. Abelev et al. [ALICE Collaboration], Exclusive J/ $\psi$ photoproduction off protons in ultra-peripheral p-Pb collisions at $\sqrt{s_{\mathrm{NN}}}=5.02 \mathrm{TeV}$, Phys. Rev. Lett. 113 (2014) no.23, 232504 doi:10.1103/PhysRevLett.113.232504 [arXiv:1406.7819 [nucl-ex]]. 
[12] R. Aaij et al. [LHCb Collaboration], Updated measurements of exclusive $J / \psi$ and $\psi(2 S)$ production cross-sections in pp collisions at $\sqrt{s}=7 \mathrm{TeV}$, J. Phys. G 41 (2014) 055002 doi:10.1088/0954-3899/41/5/055002 [arXiv:1401.3288 [hep-ex]].

[13] J. Cepila, J. G. Contreras and J. D. Tapia Takaki, Energy dependence of dissociative J/ $\psi$ photoproduction as a signature of gluon saturation at the LHC, Phys. Lett. B 766 (2017) 186 doi:10.1016/j.physletb.2016.12.063 [arXiv:1608.07559 [hep-ph]].

[14] S. P. Jones, A. D. Martin, M. G. Ryskin and T. Teubner, Probes of the small $x$ gluon via exclusive J/ $\psi$ and $\Upsilon$ production at HERA and the LHC, JHEP 1311 (2013) 085 doi:10.1007/JHEP11(2013)085 [arXiv:1307.7099 [hep-ph]].

[15] S. R. Klein, J. Nystrand, J. Seger, Y. Gorbunov and J. Butterworth, STARlight: A Monte Carlo simulation program for ultra-peripheral collisions of relativistic ions, Comput. Phys. Commun. 212 (2017) 258 doi:10.1016/j.cpc.2016.10.016 [arXiv:1607.03838 [hep-ph]].

[16] N. Armesto and A. H. Rezaeian, Exclusive vector meson production at high energies and gluon saturation, Phys. Rev. D 90 (2014) no.5, 054003 doi:10.1103/PhysRevD.90.054003 [arXiv:1402.4831 [hep-ph]].

[17] I. Bautista, A. Fernandez Tellez and M. Hentschinski, BFKL evolution and the growth with energy of exclusive $J / \psi$ and $\Upsilon$ photoproduction cross sections, Phys. Rev. D 94 (2016) no.5, 054002 doi:10.1103/PhysRevD.94.054002 [arXiv:1607.05203 [hep-ph]].

[18] J. Adam et al. [ALICE Collaboration], Coherent $\rho^{0}$ photoproduction in ultra-peripheral $\mathrm{Pb}-\mathrm{Pb}$ collisions at $\sqrt{s_{\mathrm{NN}}}=2.76 \mathrm{TeV}$, JHEP 1509 (2015) 095 doi:10.1007/JHEP09(2015)095 [arXiv:1503.09177 [nucl-ex]].

[19] V. P. Goncalves and M. V. T. Machado, Vector Meson Production in Coherent Hadronic Interactions: An update on predictions for RHIC and LHC, Phys. Rev. C 84 (2011) 011902 doi:10.1103/PhysRevC.84.011902 [arXiv:1106.3036 [hep-ph]].

[20] L. Frankfurt, M. Strikman and M. Zhalov, Signals for black body limit in coherent ultraperipheral heavy ion collisions, Phys. Lett. B 537 (2002) 51 doi:10.1016/S0370-2693(02)01882-8 [hep-ph/0204175].

[21] V. Guzey, M. Strikman and M. Zhalov, Disentangling coherent and incoherent quasielastic J/ $\psi$ photoproduction on nuclei by neutron tagging in ultraperipheral ion collisions at the LHC, Eur. Phys. J. C 74 (2014) no.7, 2942 doi:10.1140/epjc/s10052-014-2942-z [arXiv:1312.6486 [hep-ph]].

[22] E. Abbas et al. [ALICE Collaboration], Charmonium and $e^{+} e^{-}$pair photoproduction at mid-rapidity in ultra-peripheral Pb-Pb collisions at $\sqrt{s_{\mathrm{NN}}}=2.76 \mathrm{TeV}$, Eur. Phys. J. C 73 (2013) no.11, 2617 doi:10.1140/epjc/s10052-013-2617-1 [arXiv:1305.1467 [nucl-ex]].

[23] B. Abelev et al. [ALICE Collaboration], Coherent J/ $\psi$ photoproduction in ultra-peripheral $\mathrm{Pb}-\mathrm{Pb}$ collisions at $\sqrt{s_{N N}}=2.76 \mathrm{TeV}$, Phys. Lett. B 718 (2013) 1273 doi:10.1016/j.physletb.2012.11.059 [arXiv:1209.3715 [nucl-ex]].

[24] A. Adeluyi and C. A. Bertulani, Constraining Gluon Shadowing Using Photoproduction in Ultraperipheral pA and AA Collisions, Phys. Rev. C 85 (2012) 044904 doi:10.1103/PhysRevC.85.044904 [arXiv:1201.0146 [nucl-th]].

[25] S. Acharya et al. [ALICE Collaboration], Coherent $J / \psi$ photoproduction at forward rapidity in ultra-peripheral Pb-Pb collisions at $\sqrt{s_{\mathrm{NN}}}=5.02 \mathrm{TeV}$, arXiv:1904.06272 [nucl-ex]. 
[26] V. Guzey, E. Kryshen and M. Zhalov, Coherent photoproduction of vector mesons in ultraperipheral heavy ion collisions: Update for run 2 at the CERN Large Hadron Collider, Phys. Rev. C 93 (2016) no.5, 055206 doi:10.1103/PhysRevC.93.055206 [arXiv:1602.01456 [nucl-th]].

[27] V. P. Goncalves, B. D. Moreira and F. S. Navarra, Investigation of diffractive photoproduction of J/ $\psi$ in hadronic collisions, Phys. Rev. C 90 (2014) no.1, 015203 doi:10.1103/PhysRevC.90.015203 [arXiv:1405.6977 [hep-ph]].

[28] T. Lappi and H. Mantysaari, $J / \psi$ production in ultraperipheral $\mathrm{Pb}+\mathrm{Pb}$ and $p+\mathrm{Pb}$ collisions at energies available at the CERN Large Hadron Collider, Phys. Rev. C 87 (2013) no.3, 032201 doi:10.1103/PhysRevC.87.032201 [arXiv:1301.4095 [hep-ph]].

[29] A. Luszczak and W. Schäfer, Coherent photoproduction of $J / \psi$ in nucleus-nucleus collisions in the color dipole approach, Phys. Rev. C 99 (2019) no.4, 044905 doi:10.1103/PhysRevC.99.044905 [arXiv:1901.07989 [hep-ph]].

[30] J. Cepila, J. G. Contreras and M. Krelina, Coherent and incoherent J/ $\psi$ photonuclear production in an energy-dependent hot-spot model, Phys. Rev. C 97 (2018) no.2, 024901 doi:10.1103/PhysRevC.97.024901 [arXiv:1711.01855 [hep-ph]].

[31] J. Adam et al. [ALICE Collaboration], Measurement of an excess in the yield of $J / \psi$ at very low $p_{\mathrm{T}}$ in $P b$-Pb collisions at $\sqrt{s_{\mathrm{NN}}}=2.76 \mathrm{TeV}$, Phys. Rev. Lett. 116 (2016) no.22, 222301 doi:10.1103/PhysRevLett.116.222301 [arXiv:1509.08802 [nucl-ex]].

[32] J. G. Contreras, Gluon shadowing at small $x$ from coherent $\mathrm{J} / \psi$ photoproduction data at energies available at the CERN Large Hadron Collider, Phys. Rev. C 96 (2017) no.1, 015203 doi:10.1103/PhysRevC.96.015203 [arXiv:1610.03350 [nucl-ex]].

[33] J. Adam et al. [STAR Collaboration], Low- $p_{T} e^{+} e^{-}$pair production in Au+Au collisions at $\sqrt{s_{N N}}=$ $200 \mathrm{GeV}$ and $U+U$ collisions at $\sqrt{s_{N N}}=193 \mathrm{GeV}$ at STAR, Phys. Rev. Lett. 121 (2018) no.13, 132301 doi:10.1103/PhysRevLett.121.132301 [arXiv:1806.02295 [hep-ex]].

[34] M. Aaboud et al. [ATLAS Collaboration], Observation of centrality-dependent acoplanarity for muon pairs produced via two-photon scattering in Pb+Pb collisions at $\sqrt{s_{\mathrm{NN}}}=5.02 \mathrm{TeV}$ with the ATLAS detector, Phys. Rev. Lett. 121 (2018) no.21, 212301 doi:10.1103/PhysRevLett.121.212301 [arXiv:1806.08708 [nucl-ex]].

[35] Z. Citron et al., Future physics opportunities for high-density QCD at the LHC with heavy-ion and proton beams, arXiv:1812.06772 [hep-ph]. 